Conclusion In order to improve access to ART services for people with disabilities, there is need for government to develop guidelines on the management of people with disabilities and restructure data collection tools in order to capture and disaggregate data for people with disabilities accessing ART and HIV testing services.

Disclosure of interest statement This study was entirely funded by Zambia Governance Foundation and not any other partners of TALC.

\section{P17.42 SCIENTIFIC PRODUCTION OF NURSING CARE ABOUT THE CHILD BORN EXPOSED TO HIV}

ICV Lima*, VF Santos, HO Alexandre, NL Pedrosa, ODO Farias, MTG Galvão. Federal University of Ceará

\subsection{6/sextrans-2015-052270.620}

Introduction The vertical HIV transmission occurs from mother to child at the time of pregnancy, labour or postpartum. During postpartum stand out some specific care, such as: administration of chemoprophylaxis with zidovudine in the first two hours to six weeks of life; prophylaxis with trimethoprim-sulfamethoxazole from six weeks to one year; routine clinical/laboratory monitoring; systematic evaluation of the growth/development; immunisation and supply of infant milk formula. This study aimed to evaluate the scientific production of nursing care about the unborn child exposed to HIV.

Methods Integrative Review held in March 2015 in the databases: Latin American and Caribbean Literature in Health Sciences (Lilacs), Medline and Scientific Electronic Library Online (SciELO). The descriptors - Child, HIV, Vertical Transmission of Infectious Disease and Nursing - were combined using the Boolean operators AND and OR.

Results The sample consisted of nine articles in English and Portuguese published from 2006 to 2014. The categories of nursing care were addressed in articles: investigation of maternal perception of this experience; stimulating attachment between mother and child during pregnancy; encouraging verbal and nonverbal communication in maternal care actions - bathing, feeding, changing clothes; assessment of the risks of accidents and other vulnerabilities with focus in the home environment; analysis of vaccination status and awareness of the importance of immunisation; development of management actions of maternal and child care; analysis of the mother's ability to care for and empowerment. The identified care converge with the recommendations found in the literature and surpass the vertical transmission include broad actions to promote for the mother and child health.

Conclusion Research on this approach can contribute to design of nursing care proposals with a focus on providing comprehensive care to children exposed to HIV.

Disclosure of interest statement This study was funded by CAPES (Higher Education Personnel Training Coordination) and CNPq (National Council for Scientific and Technological Development). No pharmaceutical grants were received in the development of this study.

\section{P17.43 KNOWLEDGE, ATTITUDES AND COPING OF HEALTHCARE WORKERS ON HIVIAIDS AND PERSONS LIVING WITH HIVIAIDS (PLWHA) IN A TERTIARY HOSPITAL IN MANILA PHILIPPINES}

${ }^{1} \mathrm{~A}$ Lee*, ${ }^{2} \mathrm{~S}$ Bacolcol, ${ }^{3} \mathrm{~S}$ Brillantes. 'HIVIAIDS Nurse Counselor, Manila Doctors Hospital, Manila, Philippines; ${ }^{2}$ HIVIAIDS Committee Chairman, Infectious Disease Consultant, Manila Doctors Hospital, Manila, Philippines; ${ }^{3}$ Resident-in-Training, Internal Medicine, Manila Doctors Hospital, Philippines

\subsection{6/sextrans-2015-052270.621}

Introduction Starting 2006, a number of HIV cases were seen in the Philippines. It is one of nine countries with increasing cases according to USAIDS. Thus, the Filipino healthcare worker must possess accurate knowledge on the transmission, prevention and nature of HIV/AIDS, appropriate attitude and healthy coping with the demands of caring for an HIV/AIDS patient. This study determined the knowledge, attitude and coping of healthcare workers in a tertiary hospital in Manila, Philippines.

Methods A self-administered survey was used to test four concepts: socio-demographic characteristics of healthcare workers, knowledge about HIV/AIDS, attitude towards caring for HIV/ AIDS patients and coping.

Results Out of 101 respondents were 41\% males and 59\% females. Mostly are aged 20-29 years (76\%), single (83\%), registered nurses (45\%) and resident doctors-in-training (30\%). Majority were employed in the hospital for 1-5 years (55\%). Respondents' knowledge on HIV was insufficient (mean score = $16.69,76 \%$ correct answers, SD $=2.39)$. Healthcare workers scored highest on transmission and nature of the disease (78$79 \%$ ) but scored lowest on prevention (72\%). Half of respondents disagreed about stigmatising attitudes of HIV patients (45$54 \%$ ). Majority of respondents' attitudes disagreed on non-caring behaviours for HIV/AIDS patients. Coping views of healthcare workers are medium level (mean score $=3.5$ ). There was significant positive correlation between knowledge and attitude $(\mathrm{r}=0.356, \mathrm{a}=0.05):$ knowledge and coping $(\mathrm{r}=0.259, \mathrm{a}=$ $0.05)$; and attitude and coping $(\mathrm{r}=0.709, \mathrm{a}=0.05)$ of healthcare workers. There was no difference in the knowledge ( $\mathrm{P}$ two tailed $=0.3, \mathrm{p}=0.05)$, attitudes $(\mathrm{P}$ two-tailed $=0.31, \mathrm{p}=$ 0.05 ) and coping ( $P$ Two-tailed $=0.69, \mathrm{p}=0.05$ ) of respondents working for $<1$ years and $1-5$ years.

Conclusion In conclusion, there is insufficient knowledge on HIV/AIDS of healthcare workers. Implementing strategies like trainings and updates are needed to increase knowledge. Training must focus on knowledge of transmission, prevention, and nature of HIV/AIDS, could result in positive attitudes and healthy coping of healthcare workers.

\section{P17.44 AN ASSESSMENT OF HIV KNOWLEDGE AMONG TRADITIONAL BIRTH ATTENDANTS IN RURAL SETTING IN CHIPATA, ZAMBIA}

${ }^{1}$ Harry Tembo*, ${ }^{2}$ Beatrice Tembo, ${ }^{3}$ Levy Kayumbe. ${ }^{1}$ Community Advisory Board, Center for Infectious Disease Research in Zambia; ${ }^{2}$ Traditional Births Attendants Association of Zambia; ${ }^{3}$ Globe Outreach, Lusaka, Zambia

10.1136/sextrans-2015-052270.622 